\title{
Impacto en la población de la reducción de expectativas positivas sobre consumo de riesgo e intensivo de alcohol en jóvenes
}

\author{
Population impact of reducing alcohol positive \\ expectations on risky consumption and heavy \\ episodic drinking among young people
}

\author{
Lucía Moure-Rodríguez****, Carina Carbia***, Montserrat Corral**,****, Fernando \\ Cadaveira**,****, Francisco CaAmano-Isorna***. \\ * CIBER de Epidemiología y Salud Pública (CIBERESP), Departamento de Salud Pública. Universidad de Santiago de \\ Compostela, Santiago de Compostela, España. \\ ** Instituto de Investigación Sanitaria de Santiago de Compostela (IDIS). \\ *** APC Microbiome Ireland, Biosciences Building, University College Cork, Cork, Irlanda. \\ **** Departamento de Psicología Clínica y Psicobiología. Universidad de Santiago de Compostela, Santiago de Compostela, \\ España.
}

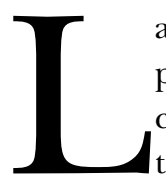

as creencias implícitas o explícitas que tiene una persona sobre las consecuencias de su propio consumo conforman las expectativas con respecto al consumo de alcohol. A pesar de referirse a las consecuencias del autoconsumo, Miller et al. nos recuerdan que las expectativas de una persona con respecto al consumo de alcohol se crean incluso antes de iniciarse el consumo real (Miller, Smith y Goldman, 1990), probablemente generadas a partir de la observación y profundamente influenciadas por las normas culturales. La tenencia de expectativas positivas sobre el consumo de alcohol se ha relacionado con mayores niveles de consumo de alcohol y el mantenimiento de los mismos, mientras que el efecto contrario se ha encontrado para las expectativas negativas (Carey, 1995; Linden, Lau- Barraco y Milletich, 2014).

Las expectativas sobre el consumo de alcohol también se han relacionado con la edad de inicio (Janssen, Treloar, Merrill y Jackson, 2018), una variable de alto interés, relacionada con el consumo de alcohol durante la juventud e incluso con las consecuencias negativas de este consumo.

Estamos realizando un estudio de cohorte de 12 años de seguimiento entre estudiantes universitarios (Cohorte Compostela, España) desde el 2005 para identificar predictores y prevalencia del consumo de alcohol entre estudian- tes universitarios $(\mathrm{N}=1382)$. Este estudio fue aprobado por el Comité de Bioética de la Universidad de Santiago de Compostela. El estudio Cohorte Compostela ha demostrado que algunas personas comienzan la universidad sin haber consumido alcohol, mientras que otras ya participan en Consumo de Riesgo (CR) o Consumo Episódico Excesivo (CEE). Además, entre los estudiantes de la Cohorte Compostela, las expectativas de consumo de alcohol a los 17-18 años de edad nos ha permitido predecir sus patrones $\mathrm{y}$ tendencias de consumo, incluso para el seguimiento de 9 años. Teniendo en cuenta estos resultados, nuestro objetivo es determinar el impacto de la reducción de las expectativas positivas respecto al alcohol sobre el CR y el CEE.

Se midieron el CR (variable dicotómica generada a partir de la puntuación en el AUDIT) y el CEE (variable dicotómica generada a partir de la tercera pregunta en el AUDIT) (Varela, Carrera, Rial, Braña y Osorio, 2006), además de otros factores asociados al consumo de alcohol (nivel de estudios y consumo de los padres, edad de inicio del consumo y las expectativas respecto del consumo de alcohol, entre otros). En cuanto a las expectativas respecto del consumo de alcohol, los estudiantes tuvieron que clasificar 7 expectativas positivas y 7 expectativas negativas en relación con los efectos del alcohol (Defensor del Menor

Recibido: Diciembre 2020; Aceptado: Febrero 2021.

Enviar correspondencia a:

Lucía Moure Rodríguez.

E-mail: lucia.moure.rodriguez@usc.es 
de la Comunidad de Madrid, 2002). Teniendo en cuenta el número de expectativas positivas y negativas, se generó una puntuación entre 0 y 14 (siendo 0 el máximo de expectativas negativas y 14 el máximo de expectativas positivas). Las puntuaciones se dividieron en terciles.

Para determinar el impacto de la reducción de las expectativas positivas respecto al alcohol sobre el CR y el CEE, realizamos un nuevo análisis para calcular la Fracción Atribuible Poblacional (FAP) para ambas variables. La FAP nos permite calcular la carga de las expectativas en la práctica de CR y CEE en los estudiantes universitarios, y, por tanto, qué efecto tendría la reducción de estas expectativas en ambos patrones de consumo. Utilizamos la fórmula propuesta por Morgenstern y Bursic (1982), porque permite hacer cálculos basados en efectos ajustados para otras variables y, por tanto, con menos sesgo. Además, esta fórmula va más allá del cálculo clásico que da por hecho que el factor de riesgo desaparecerá de la población en estudio, y permite hacer estimaciones de la reducción de estas conductas en escenarios en los que cambia la distribución del factor de riesgo en estudio y el objetivo de la intervención, con una menor exposición en la población, pero sin desaparecer. Por tanto, proporciona cálculos más aplicables a la realidad. Para más detalles sobre este método, véase Caamano-Isorna, Adkins, Aliev, Moure-Rodríguez y Dick, 2020. Tuvimos en cuenta riesgos relativos ajustados para las expectativas positivas respecto al alcohol, las tasas de prevalencia del consumo y la proporción de bebedores de cada nivel de expectativas de Moure-Rodríguez et al. 2016.

Las FAP para las expectativas altamente positivas respecto al alcohol en CR y CEE fueron respectivamente $64,6 \%$ y $56,7 \%$ en mujeres y $49,4 \%$ y $48,0 \%$ en hombres. Dado que no sería realista asumir que todos los jóvenes reducen por completo sus expectativas positivas respecto al alcohol, también se calculó el porcentaje de CR y CEE que se eliminaría si los adolescentes redujesen parcialmente sus expectativas respecto al alcohol (Figura 1). Como resultado, obtuvimos figuras representativas del efecto que las intervenciones poblacionales tendrían en la reducción de las expectativas positivas entre los jóvenes con diferentes niveles de eficacia. Estos cálculos son de gran interés para la toma de decisiones en salud pública y su uso es cada vez más común porque nos permiten transformar aproximaciones teóricas en realidad.

Se sabe que los jóvenes cambian sus expectativas respecto del consumo de alcohol a lo largo de la adolescencia, aumentando las expectativas positivas a medida que pasan los años (Copeland, Proctor, Terlecki, Kulesza y Williamson, 2014). Nuestros resultados sugieren que podríamos reducir considerablemente estas conductas de riesgo entre los estudiantes universitarios a través de intervenciones enfocadas en evitar este aumento de expectativas positivas y trabajar el mantenimiento de expectativas negativas desde una edad temprana.

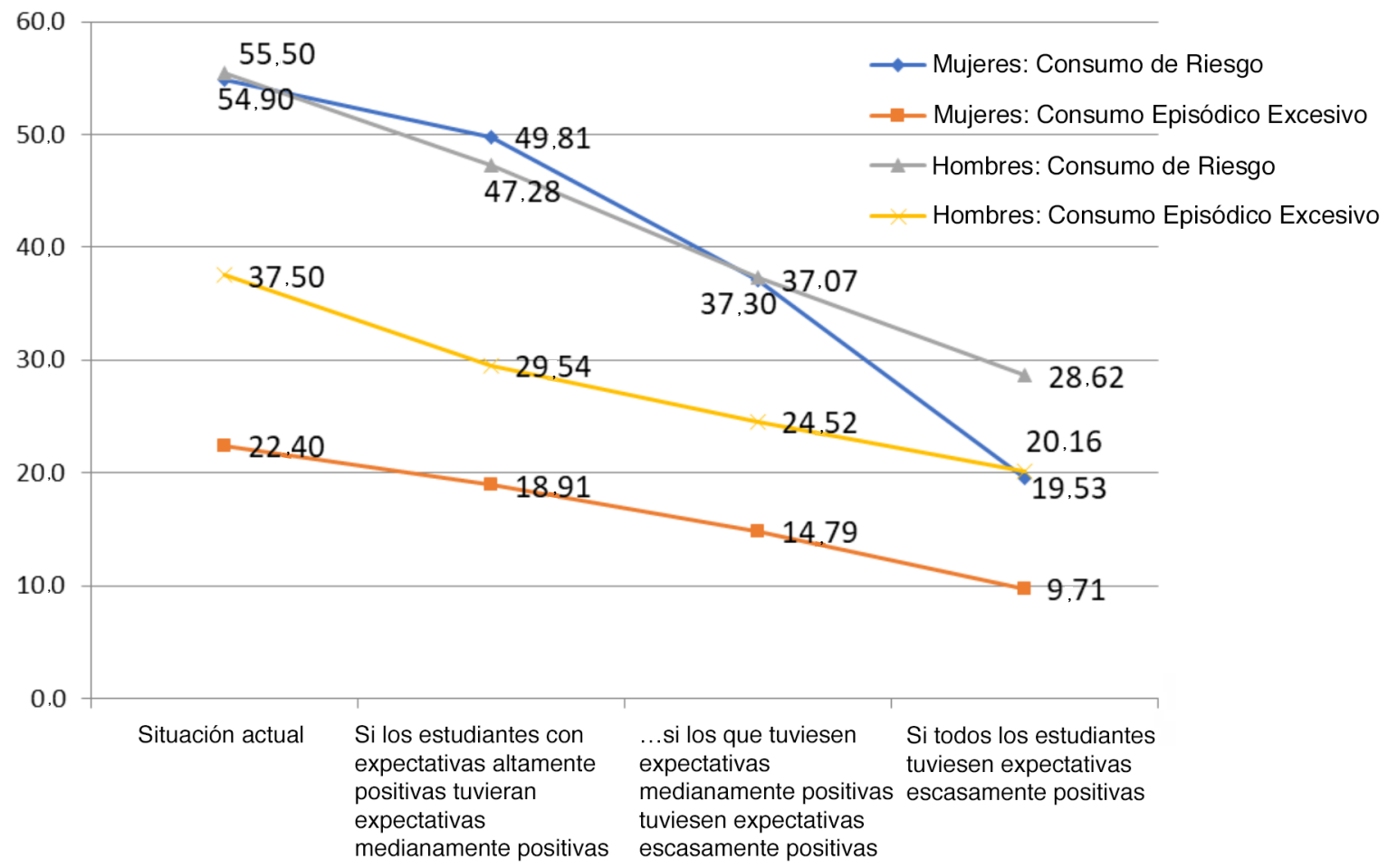

Figura 1. Impacto estimado de la reducción de las expectativas positivas respecto al alcohol en la prevalencia (\%) del consumo de riesgo y el consumo episódico excesivo. 


\section{Reconocimientos}

Este trabajo ha sido financiado por el Plan Nacional sobre Drogas (España) (2005/PN014) y el Fondo de Investigación en Salud (España) (PI15/00165). Carina Carbia ha sido financiada por el programa de investigación e innovación Horizonte 2020 de la Unión Europea con la beca Marie Sklodowska-Curie No. 754535.

\section{Conflicto de intereses}

Los autores declaran la inexistencia de conflicto de interés.

\section{Referencias}

Caamano-Isorna, F., Adkins, A., Aliev, F., Moure-Rodríguez, L. y Dick, D. M. (2020). Population Attributable fraction of early age of onset of alcohol use in alcohol abuse and dependence: A 3-year follow-up study in university students. International Journal of Environmental Research and Public Health, 17, 2159. doi:10.3390/ijerph17062159.

Carey, K. B. (1995). Alcohol-related expectancies predict quantity and frequency of heavy drinking among college students. Psychology of Addictive Behaviors, 9, 236-241. doi:10.1037/0893-164X.9.4.236.

Copeland, A. L., Proctor, S. L., Terlecki, M. A., Kulesza, M. y Williamson, D. A. (2014). Do positive alcohol expectancies have a critical developmental period in pre-adolescents? Journal of Studies on Alcohol and Drugs, 75, 945952. doi:10.15288 / jsad.2014.75.945.

Defensor del Menor de la Comunidad de Madrid. (2002). Analysis of the alcohol consumption among youths of Madrid Community. En Defensor del Menor de la Comunidad de Madrid: Estudios e Investigaciones 2002. Madrid: Defensor del Menor en la Comunidad de Madrid; 2002. pp. 307-398.

Janssen, T., Treloar, H., Merrill, J. E. y Jackson, K. M. (2018). Developmental relations between alcohol expectancies and social norms in predicting alcohol onset. Developmental Psychology, 54, 281-292. doi:10.1037/dev0000430.

Linden, A. N., Lau-Barraco, C. y Milletich, R. J. (2014). Protective behavioral strategies, alcohol expectancies, and drinking motives in a model of college student drinking. Psychology of Addictive Behaviors, 28, 952-959. doi:10.1037/a0037041.

Miller, P. M., Smith, G. T. y Goldman, M. S. (1990). Emergence of alcohol expectancies in childhood: A possible critical period. Journal of Studies on Alcohol, 51, 343-349. doi:10.15288/jsa.1990.51.343.

Morgenstern, H. y Bursic, E. S. (1982). A method for using epidemiologic data to estimate the potential impact of an intervention on the health status of a target popula- tion. Journal Community Health, 7, 292-309. doi:10.1007/ BF01318961.

Moure-Rodríguez, L., Piñeiro, M., Corral, M., Rodríguez-Holguín, S., Cadaveira, F. y Caamaño-Isorna, F. (2016). Identifying predictors and prevalence of alcohol consumption among university students: Nine years of follow-up. PLoS One, 11, e0165514. doi:10.1371/journal. pone.0165514.

Varela, J., Carrera, A., Rial, A., Braña, T. y Osorio, J. (2006). Identificación dos trastornos debidos ao consumo de alcohol. Unha adaptación do AUDIT á poboación galega [Validation of AUDIT for Galician population]; Xunta de Galicia-Consellería de Sanidade-Sergas: Santiago de Compostela, Spain. 
